\title{
Control of the Spin Wave Phase in Transmission through the Ultrathin Interface between Exchange Coupled Ferromagnetic Materials
}

\author{
M. Mailian ${ }^{a, *}$, O.Y. Gorobets ${ }^{a, b}$, Y.I. Gorobets ${ }^{b}$, M. Zelent ${ }^{c}$ And M. KrawczyK ${ }^{c}$ \\ ${ }^{a}$ Faculty of Physics and Mathematics, National Technical University of Ukraine "Igor Sikorsky Kyiv Polytechnic \\ Institute", 37 Peremogy ave., 03056, Kyiv, Ukraine \\ ${ }^{b}$ Institute of Magnetism, National Academy of Sciences of Ukraine, 36-b Vernadskogo st., 03142, Kyiv, Ukraine \\ ${ }^{c}$ Faculty of Physics, Adam Mickiewicz University in Poznan, Umultowska 85, Poznań, 61-614, Poland
}

\begin{abstract}
In this paper, we show analytically and numerically, that the ultrathin metallic layer up to few monatomic layers separating two ferromagnetic media, which are exchange coupled, can effectively change the phase of the reflected or transmitted spin waves. Taking into account ground states with parallel orientations of the magnetization vectors, we developed the analytical model, which shows the possibility of spin-wave phase control by varying the exchange coupling strength between two ferromagnets. Moreover, we demonstrate with micromagnetic simulations that the effect still exists for the spin waves propagating in thin ferromagnetic film during the reflection or transmission from the interface, where interlayer exchange interactions are present.
\end{abstract}

DOI: 10.12693/APhysPolA.133.480

PACS/topics: 75.30.Ds, 75.30.Kz, 75.78.Cd

\section{Introduction}

The spin-wave (SW) phase shifters attract much attention of researchers. Development of a new generation of logic devices encoding information in the phase of travelling SW packets and utilizing them for data processing is of great interest [1]. The methods of the phase shifting based on externally initiated change of the local effective magnetic field for changing the dispersion relation can be achieved with the SW travelling through a noncollinear structures like domain walls [2], with applying a local spin-polarized current [3], applying local voltage [4] or the local Oersted fields [1]. The methods involving electrical current have limitations due to Joule heating and electrical power consumption. At the same time, the nucleation and motion of domain walls are energetically costly and difficult to control precisely. The control of SW phase shift can be realized using the intrinsic nonlinear features of the magnetization oscillation allowing for the conversion of the SW phase into a SW intensity and vice-versa [5]. With this purpose, we investigate the SW propagation through the specifically designed interface which ensures exchange coupling between two ferromagnetic materials. Such interface can be realized in various ways, e.g., by an ultrathin non-magnetic metallic spacer separating materials, allowing for the RudermanKittel-Kasuya-Yosida (RKKY) coupling [6, 7]. In this case the thickness of the spacer should be much shorter than the wavelength of the operating wave to control the SW phase.

*corresponding author; e-mail: marina92mmd@gmail.com

\section{Methods}

\subsection{Analytical model}

We consider the system of two semi-infinite ferromagnetic media, separated by a thin layer of non-magnetic material (the spacer) in the uniform constant external magnetic field $\boldsymbol{H}_{0}$ parallel to the axis $O z$ (see Fig. 1). The axis $O x$ is chosen along the normal to the interface between the ferromagnetic materials with the interface centered at $x=0$. In the analytical model we include the exchange interaction, uniaxial anisotropy and the static magnetic field. Magnetostatic interactions and damping are neglected here. The total energy density of such a system can be written generally in the following form: [8]

$$
w=\sum_{j=1}^{2} \theta\left((-1)^{j} x\right) w_{j}+\delta(x) \cdot w_{0}\left(\boldsymbol{M}_{1}, \boldsymbol{M}_{2}\right)
$$

which is suitable to apply to the system of two ferromagnets with the finite thickness of the interface between them [9]. In Eq. (1), $\theta(x)$ is the Heaviside step function, $\delta(x)$ is the Dirac delta function, and $j$ indexes the two ferromagnetic media $(j=1,2)$. The first term on the right hand of Eq. (1) describes the magnetic energy density of the bulk material:

$$
w_{j}=\frac{\alpha_{j}}{2}\left(\frac{\partial \boldsymbol{M}_{j}}{\partial x}\right)^{2}-\frac{1}{2} \beta_{j}\left(\boldsymbol{M}_{j} \boldsymbol{n}_{j}\right)^{2}-\boldsymbol{H}_{0} \boldsymbol{M}_{j} .
$$

The three terms on the right hand side of Eq. (2) are exchange energy density, anisotropy energy density and Zeeman energy density, respectively. $\boldsymbol{M}_{j}$ is the magnetization vector in the $j$-th media, $\alpha_{j}=2 \pi l_{e x}^{2}=A_{e x j} / M_{0 j}^{2}$, where $l_{e x}$ is the exchange length of the $j$-th ferromagnet and $A_{e x j}$ is the $j$-th ferromagnet exchange stiffness constant, $\beta_{j}=K_{j} / M_{0 j}^{2}$ with $K_{j}$ being uniaxial magnetic 
anisotropy constant, $\boldsymbol{n}_{j}$ is the unit vector pointing in the direction of the easy axis in the $j$-th ferromagnet. The second term in Eq. (1) denotes the surface energy density at the interface:

$$
w_{0}=A_{12} M_{1} M_{2} \text {. }
$$

The term $A_{12} M_{1} M_{2}$ describes energy per unit area of the interlayer exchange coupling between magnetic layers mediated by a non-magnetic spacer, with interlayer exchange constant $A_{12}$. The interlayer exchange constant can have different origin, including RKKY interactions.

The Landau-Lifshitz equation for each ferromagnet has the following form in the case when dissipation is neglected:

$$
\frac{\partial \boldsymbol{M}_{j}}{\partial t}=\gamma \boldsymbol{M}_{j} \times \boldsymbol{H}_{e f f}^{j},
$$

where $\gamma$ is the gyromagnetic ratio. The effective magnetic field $\boldsymbol{H}_{\text {eff }}^{j}$ is defined as:

$$
\boldsymbol{H}_{e f f}^{j}=-\frac{\delta W}{\delta \boldsymbol{M}_{j}},
$$

where $W=\int_{V} w \mathrm{~d} V$ denotes the total magnetic energy of the system ( $V$ is a volume of the whole system). The formula for the final components of the effective magnetic field entering Eq. (4) can be found in Ref. [9]. In linear approximation and assuming plane wave harmonic solutions with wave vector $\boldsymbol{k}_{j}$ and angular frequency $\omega_{j}$ for the dynamical components of the magnetization vector, the dispersion law for each of the ferromagnets from the Landau-Lifshitz Eq. (4) can be written as follows (see graphical dependence in the inset to the Fig. 2):

$$
\omega_{j}\left(\boldsymbol{k}_{j}\right)=\gamma\left(H_{0}+\beta_{j} M_{0 j}+\alpha_{j} M_{0 j} k_{j}^{2}\right) \text {. }
$$

We define the boundary conditions for the magnetization vector at the interface to obtain amplitudes and phase shifts of the reflected and transmitted waves [9]:

$$
\begin{aligned}
& \left.\left(A_{12} m_{2 n}+C \xi m_{1 n}+\alpha_{1} \frac{\partial m_{1 n}}{\partial x}\right)\right|_{x=0}=0 \\
& \left.\left(A_{12} m_{1 n}+\frac{C}{\xi} m_{2 n}-\alpha_{2} \frac{\partial m_{2 n}}{\partial x}\right)\right|_{x=0}=0
\end{aligned}
$$

where $n=x, y, C=-A_{12} \operatorname{sgn}\left(M_{1} M_{2}\right), \xi=M_{02} / M_{01}$, $\operatorname{sgn}\left(M_{1} M_{2}\right)=\left[\begin{array}{rr}1, & \left(M_{1} M_{2}\right)>0 \\ -1, & \left(M_{1} M_{2}\right)<0\end{array}\right.$ is a signum function. In the following we will consider only ferromagnetic arrangement of the magnetizations $\boldsymbol{M}_{1}$ and $\boldsymbol{M}_{2}$, i.e., we assume $C=-A_{12}$. We have assumed here, that the interlayer thickness is much smaller than the SW wavelength and thus the interface has been reduced to the plane of zero thickness, positioned at $x=0$. The details can be found in Ref. [10].

Using the dispersion relations for each ferromagnetic material Eq. (6), and taking into account the equality of frequencies $\omega=\omega_{1}=\omega_{2}$ for monochromatic wave we can rewrite components $k_{1}$ and $k_{2}$ as follows:

$$
k_{j}=\sqrt{\left(\left(\omega / \gamma-H_{0}\right) / \alpha_{j} M_{0 j}-\beta_{j} / \alpha_{j}\right)} .
$$

The dynamical components of the magnetization vector in the first $(F M(-) 1)$ and the second $(F M(-) 2)$ ferro- magnetic material can be written as

$$
\begin{aligned}
& m_{1 x}+\mathrm{i} m_{1 y}=I_{1} \exp \left(\mathrm{i}\left(k_{1} x-\omega t-\varphi_{1}\right)\right) \\
& \quad+I_{1 R} \exp \left(\mathrm{i}\left(k_{1} x-\omega t+\varphi_{1 R}\right)\right), \\
& m_{2 x}+\mathrm{i} m_{2 y}=I_{T} \exp \left(\mathrm{i}\left(k_{2} x-\omega t\right)\right),
\end{aligned}
$$

where $I_{1}, I_{1 R}, I_{T}$ are the amplitudes of the incident, reflected and refracted waves, respectively. Due to the symmetry of the structure, circular precession of the magnetization is assumed in solutions (9). We also allowed, that the phase of the wave during transmission and reflection can change, however the change of the phase will be assumed as independent on the position, i.e. phases $\varphi_{1}$ and $\varphi_{1 R}$ are constants. Note, that we have assumed as a reference phase the phase of the transmitted wave.

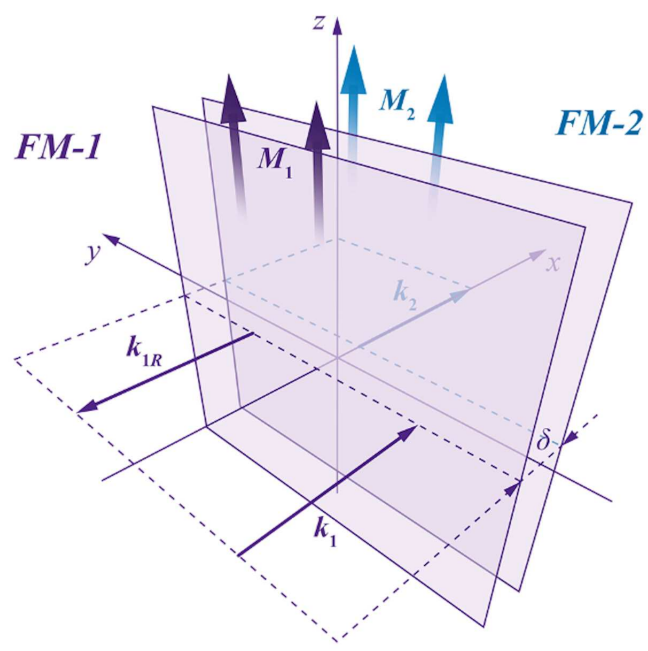

Fig. 1. Schematic plot of the two semi-infinite ferromagnetic media $F M(-) 1$ and $F M(-) 2$ separated by the non-magnetic thin film of thickness $\delta$. SW incidences normally with the wave vectors of the incident, reflected and transmitted SWs: $\boldsymbol{k}_{1}, \boldsymbol{k}_{1 R}$ and $\boldsymbol{k}_{2}$, respectively.

Implementation of the Eqs. (9) to the boundary conditions (7) gives the phase shifts, reflection and transmission coefficients of the SW in the following form:

$$
\begin{aligned}
& \operatorname{ctg}\left(\varphi_{1,1 R}\right)=\frac{A_{12}\left(\xi \alpha_{2} k_{2} \pm \alpha_{1} k_{1} / \xi\right)}{\alpha_{1} \alpha_{2} k_{1} k_{2}}, \\
& R=\sqrt{\frac{\left(\alpha_{1} \alpha_{2} k_{1} k_{2}\right)^{2}+A_{12}^{2}\left(\xi \alpha_{2} k_{2}-\alpha_{1} k_{1} / \xi\right)^{2}}{\left(\alpha_{1} \alpha_{2} k_{1} k_{2}\right)^{2}+A_{12}^{2}\left(\xi \alpha_{2} k_{2}+\alpha_{1} k_{1} / \xi\right)^{2}}} \\
& T=\frac{2 \alpha_{1} k_{1}}{\sqrt{\left(\alpha_{1} \alpha_{2} k_{1} k_{2} / A_{12}\right)^{2}+\left(\xi \alpha_{2} k_{2}+\alpha_{1} k_{1} / \xi\right)^{2}}} .
\end{aligned}
$$

where the reflection coefficient $R=I_{1 R} / I_{1}$ and the transmission coefficient $T=I_{T} / I_{1}$.

\subsection{Micromagnetic simulations}

The micromagnetic simulations (MSs) were performed with MuMax [11] for the thin film geometry (thickness 
$10 \mathrm{~nm}$ ) with the dipolar interactions and damping neglected. The interface has been implemented as single discretization cell $\Delta$ with effective exchange coupling related to $A_{12}$ parameter from the analytical model.

\section{Results}

We perform study of the phase shift for the transmitted SW in dependence on the interlayer exchange coupling $A_{12}$. To compare the results from two models the $A_{12}$ has been normalized to the dimensionless exchange parameter using the equation $A_{12}^{S}=A_{12} \cdot\left(-\Delta \cdot M_{\mathrm{Co}}^{2} / 2 A_{e x \mathrm{Co}}\right)$, where $\Delta=0.5 \mathrm{~nm}$ is a single discretization cell, $M_{\mathrm{Co}}$ and $A_{\text {ex } \mathrm{Co}}$ are, respectively, the saturation magnetization and the exchange stiffness constant of cobalt. The results for the system composed of cobalt - non-magnetic coupling layer - cobalt are shown in Fig. 2. We assume the static external magnetic field $H_{0}=20 \mathrm{kOe}$.

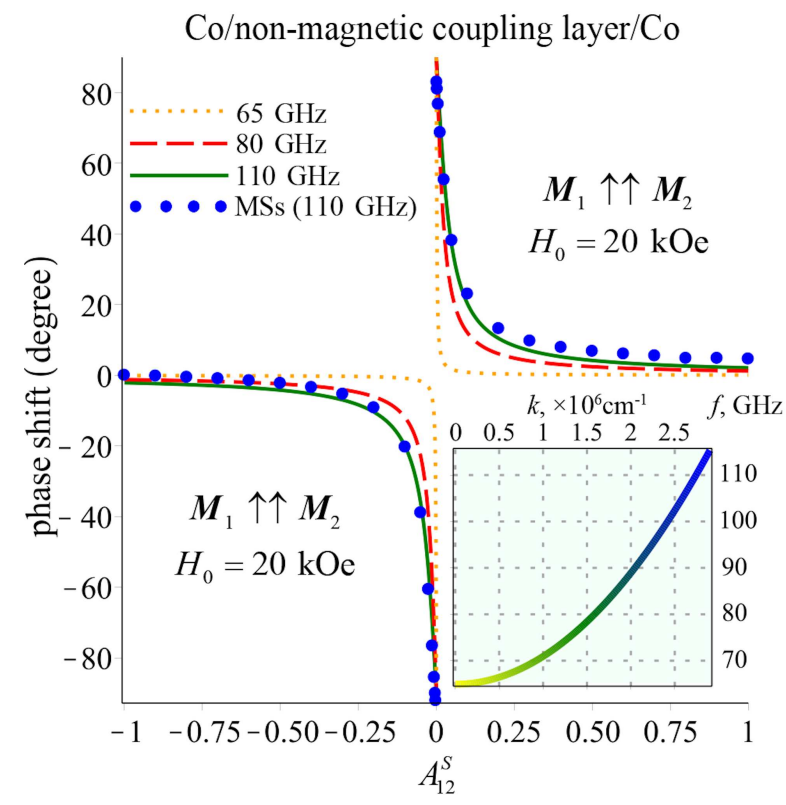

Fig. 2. The dependences of the phase shift of the transmitted SW on the normalized interlayer exchange coupling parameter $A_{12}^{S}$ for the ferromagnetic ground state $M_{1} \uparrow \uparrow M_{2}$ in the system Co/non-magnetic coupling layer/Co. The inset to the figure is the graphical dependence of frequency $f$ on the wave vector $\boldsymbol{k}$ for Co.

The following material parameters are used for calculation: $K_{1}=K_{2}=K_{\mathrm{Co}}=4.5 \times 10^{6} \mathrm{erg} \mathrm{cm}^{-3}$ [12], $M_{01}=$ $M_{02}=M_{\mathrm{Co}}=1.4 \times 10^{3} \mathrm{G}[13], A_{\text {ex } \mathrm{Co}}=3 \times 10^{-6} \mathrm{erg} / \mathrm{cm}$, $\alpha_{1}=\alpha_{2}=\alpha_{\text {Co }}=1.43 \times 10^{-12} \mathrm{~cm}^{2}$ [13]. The analytical results have been obtained for different frequency values: $65 \mathrm{GHz}$ (orange dotted lines), $80 \mathrm{GHz}$ (red dashed lines) and $110 \mathrm{GHz}$ (green solid lines). The MSs data for $110 \mathrm{GHz}$ (blue dots) match with the analytical results, which validates the analytical approach. We found that for $A_{12}^{S}<0$ the SW has negative phase shift in transmission decreasing from 0 for $A_{12}^{S}=-1$ (i.e., continuous Co film) to $\varphi_{1}=-90^{\circ}$ for $A_{12}^{S}=0$. For the positive exchange coupling the $\mathrm{SW}$ is delayed in transmission $\left(\varphi_{1}>0\right)$ with the phase varied from $90^{\circ}$ to 0 .

\section{Conclusions}

In this paper we demonstrate a new possibility to introduce a controlled phase shift in a broad range of the propagating SWs. This is achieved in the transmission of SWs through an ultrathin nonmagnetic spacer introducing exchange coupling between the two ferromagnetic materials. The proposed mechanism of the SW phase shifting can be further exploited for the designing metasurfaces for SWs.

\section{Acknowledgments}

This research has received founding from EU Horizon2020 research and innovation program under the Marie Sklodowska-Curie grant agreement 644348 (MagIC) and from the National Science Centre Poland grant UMO-2012/07/E/ST3/00538.

\section{References}

[1] K.S. Lee, S.K. Kim, J. Appl. Phys. 104, 053909 (2008).

[2] R. Hertel, W. Wulfhekel, J. Kirschner, Phys. Rev. Lett. 93, 257202 (2004).

[3] X. Chen, Q. Wang, Y. Liao, X. Tang, H. Zhang, Z. Zhong, Journal of Magnetism and Magnetic Materials 394, 67(2015).

[4] A. Khitun, K.L. Wang, J. Appl. Phys. 110, 034306 (2011).

[5] T. Brächer, F. Heussner, P. Pirro, T. Meyer, T. Fischer, M. Geilen, B. Heinz, B. Lägel, A.A. Serga, B. Hillebrands, Scientific Reports 6, 38235 (2016).

[6] M.A. Ruderman, C. Kittel, Phys. Rev. 96, 99 (1954).

[7] P. Bruno, C. Chappert, Phys. Rev. B 46, 261 (1992).

[8] Yu.I. Gorobets, V.I. Finokhin, Phys. Metal. Metal. 56, 226 (1983).

[9] V.V. Kruglyak, O.Yu. Gorobets, Yu.I. Gorobets, A.N. Kuchko, J. Phys.: Condens. Matter 26, 406001 (2014).

[10] P. Gruszecki, M. Mailyan, O. Gorobets, M. Krawczyk, Phys. Rev. B 95, 014421 (2017).

[11] A. Vansteenkiste, J. Leliaert, M. Dvornik, M. Helsen, F. Garcia-Sanchez, B. Van Waeyenberge, AIP Advanced 4, 107133 (2014).

[12] B.D. Cullity, C.D. Graham, Introduction to Magnetic Materials, John Wiley, Hoboken 2005.

[13] W. Chen, M. Han, Piers Online. 6, 101 (2010). 